\title{
Levosimendan in the modern treatment of patients with acute heart failure of various aetiologies
}

Lidia Glinka ${ }^{1}$, Ewa Mayzner-Zawadzka ${ }^{1}$, Dariusz Onichimowski ${ }^{2}$, Rakesh Jalali ${ }^{3}$, Maciej Glinka ${ }^{4}$

\author{
${ }^{1} 2^{\text {nd }}$ Anaesthesiology and Intensive Care Clinical Ward, Clinical University \\ Hospital, Department of Anaesthesiology and Intensive Care, University of Warmia \\ and Mazury, Olsztyn, Poland \\ ${ }^{2} 1^{\text {st }}$ Clinical Department of Anaesthesiology and Intensive Care, Regional Specialist \\ Hospital, Olsztyn, Poland \\ ${ }^{3}$ Emergency Department, Regional Specialist Hospital, Olsztyn, Poland \\ ${ }^{4}$ Department of Cardiology, Regional Specialist Hospital, Olsztyn, Poland
}

Submitted: 4 October 2017; Accepted: 29 November 2017;

Online publication: 7 November 2019

Arch Med Sci 2021; 17 (2): 296-303

DOI: https://doi.org/10.5114/aoms.2018.77055

Copyright $\odot 2019$ Termedia \& Banach

\section{Abstract}

Acute decompensated heart failure (ADHF) is a common clinical problem associated with a high mortality rate. Because ADHF has various aetiologies, there are a range of therapeutic options, among others, positive inotropes (inotropic drugs). As an inotropic agent whose mechanism is different than that of "classical" medicines, levosimendan (LSM) is one of the most common therapeutic options. Despite many publications on LSM, some issues related to its application remain unclear. The authors of this paper have attempted to summarise expert recommendations and reports available in the literature.

Key words: acute decompensated heart failure, inotropic drugs, levosimendan.

\section{Introduction}

The growing number of patients with circulation disorders forces researchers to develop new effective medicines. Heart failure is one of the most common civilisational diseases in the modern world. Irrespective of its aetiology, when its symptoms grow more acute, the condition poses a threat to patients' lives and often requires the introduction of inotropic drugs [1]. In the recent years, levosimendan (LSM) has been used more and more often as a novel therapeutic option.

\section{General overview and mechanism of levosimendan}

Levosimendan was introduced onto the pharmaceutical market in 2000 in Sweden. In Poland, its availability was limited until 2015 because it was available only as a direct import. Since LSM was included as a drug available in hospital conditions, its application has considerably increased. There have also appeared many reports analysing its use in clinical conditions.

Levosimendan is a positive inotropic drug. LSM acts according to a different mechanism. Its singularity consists of increasing the heart's contractility without increasing oxygen consumption. In the long run, this results in a lower mortality rate in comparison with dobutamine or

\author{
Corresponding author: \\ Lidia Glinka MD, PhD \\ $2^{\text {nd }}$ Anaesthesiology and \\ Intensive Care Clinical Ward \\ Clinical University Hospital \\ 30 Warszawska St \\ 10-082 Olsztyn, Poland \\ Phone/fax: +48 895245308 \\ Fax: +48 895413093 \\ E-mail: lidka.glinka@ \\ gmail.com
}


milrinone therapies. LSM binds troponin C and increases its sensitivity to calcium ions (it is a socalled calcium sensitiser) [2]. This increases the strength of myofilament contraction with no need for an energy-consuming rise of calcium ion concentration in the cell. Additionally, the drug opens ATP-dependent potassium channels in vascular smooth muscles, which leads to dilatation of veins and arteries (including coronary ones). As a result, both preload and afterload decrease, the coronary flow improves, and the visceral flow improves. The third mechanism of LSM activity consists of opening ATP-dependent potassium channels in mitochondria. This results in a better use of energy in the cell, which increases its resistance to oxygen deficiency. This effect was observed in heart cells, brain cells, and liver cells. A better use of energy results in lower production of free radicals and their higher utilisation. This in turn limits cell damage and stimulation of production of inflammatory response mediators $[3,4]$.

Application of LSM results in: increased cardiac output (CO), increased stroke volume (SV), decreased pulmonary capillary wedge pressure (PCWP), decreased pulmonary vascular resistance (PVR), decreased mean pulmonary artery pressure (MPAP), and decreased systemic vascular resistance (SVR). This favourable haemodynamic profile leads to limitation or disappearance of acute decompensated heart failure (ADHF) symptoms, resulting in a better oxygen supply to the cells and increased blood flow through body organs $[5,6]$.

The recommended dosage of LSM is from 0.05 to $0.2 \mathrm{mg} / \mathrm{kg} / \mathrm{min}$ in a 24 -hour infusion. The pharmacokinetic properties of LSM are linear when it comes to therapeutic doses; the drug is almost completely (94\%) bound with glutathione (non-active) and excreted with urine and faeces. About $6 \%$ of non-metabolised drug is reduced to OR 1855 and then acetylated to OR 1896 in the intestines. The process of acetylation is influenced by the intestinal flora. The half-life of the drug in the final stage of elimination is about $1 \mathrm{~h}$, but the active metabolites act for 14 days more. The effect of a 24-hour intravenous infusion persists for a considerable period of time [7]. The peak of LSM activity appears between the sixth and ninth hour of the infusion, which is why some clinicians decide to administer the drug once again within $48 \mathrm{~h}$, in order for its maximal effect to appear in the greatest decompensation phase, for instance during cardiac surgical procedures [8].

The second dosage regimen includes an intravenous infusion after a 10-minute dose of 6-12 $\mathrm{kg} / \mathrm{kg}$. This is not recommended because of considerable reduction in blood pressure (BP) caused by an abrupt decrease in SVR. It is recommended that LSM be administered carefully in patients with a low BP at baseline. Most of the examined patients were initially given LSM combined with noradrenaline, which was discontinued when haemodynamic parameters improved [8].

\section{Comparison to other drugs used in acute decompensated heart failure}

Classical inotropic drugs act most often through $\alpha$ and $\beta$ receptors of the heart and blood vessels, increasing the level of cyclic adenosine monophosphate (CAMP) and intracellular calcium concentration (dobutamine, adrenaline, dopamine). Phosphodiesterase inhibitors (amrinone, milrinone) prevent CAMP degradation. All these activities are aimed at increasing the force of myocardial contraction, and they influence the preload and afterload of the heart. However, they show some side effects, the most serious of which are increased myocardial oxygen consumption and arrhythmia (Table I) [9, 10].

\section{Levosimendan in acute decompensated heart failure}

Acute decompensated heart failure was one of the first indications to administer LSM. Because the number of patients undergoing LSM treatment was growing, studies on applying LSM in the following cases appeared: 1) perioperative ther-

Table I. Comparison of action of inotropic drugs

\begin{tabular}{|c|c|c|c|c|c|c|c|}
\hline Inotropic drug & $\mathrm{CO}$ & SVR & PVR & PCWP & $\begin{array}{l}\text { Myocardium } \\
\text { oxygen } \\
\text { consumption }\end{array}$ & Tachyphylaxis & $\begin{array}{l}\text { Receptors/ } \\
\text { mechanism }\end{array}$ \\
\hline Epinephrine & $\uparrow \uparrow$ & $\uparrow$ in high doses & $\uparrow$ & $\uparrow$ & $\uparrow$ & No & $\beta 1, \beta 2, \alpha 1$ \\
\hline Dopamine & $\uparrow$ & $\uparrow$ & $\uparrow$ & $\uparrow$ & $\uparrow$ & Yes & $\mathrm{D} 1, \alpha 1, \beta 1$ \\
\hline Dobutamine & $\uparrow$ & $\downarrow$ & $\downarrow$ & $\downarrow$ & $\uparrow \uparrow$ & Yes & $\beta 1, \beta 2, \alpha 1$ \\
\hline Levosimendan & $\uparrow$ & $\downarrow$ & $\downarrow$ & $\downarrow$ & No & No & $\uparrow \mathrm{Ca}^{+}=\uparrow \mathrm{cAMP}$ \\
\hline Milrinone & $\uparrow$ & $\downarrow$ & $\downarrow$ & $\downarrow$ & $\uparrow$ & No data & PDE inh. $=\uparrow \mathrm{cAMP}$ \\
\hline Norepinephrine & $\uparrow$ & $\uparrow$ & $\uparrow$ & $\uparrow$ & $\uparrow$ & Yes & $\alpha 1, \beta 1$ \\
\hline
\end{tabular}


apy in cardiac surgery, 2) right ventricle failure, 3) peripartum cardiomyopathy, 4) stunned myocardium, 5) intoxication with calcium channel blockers (CCBs), and 6) septic shock.

The common denominator of all these conditions is ADHF, often assuming the form of cardiogenic shock with the accompanying low cardiac output syndrome.

As is commonly known, classical ADHF treatment is based on improving $\mathrm{CO}$ and consequently improving oxygen supply to the cells. This is achieved by means of treating the cause (cardiac surgical procedures), optimising intravascular volume status (with diuretic agents, renal replacement techniques, intravascular volume expansion), and administering inotropic drugs, and others [1].

Levosimendan demonstrates advantages that were successfully used in ADHF treatment. LSM improves haemodynamic parameters without considerable increase in oxygen consumption, it diminishes ADHF symptoms, and its activity persists thanks to the origination of an active metabolite. It also provides additional benefits for patients treated with $\beta$-adrenolytic drugs. In the literature there are a number of comparative studies analysing the effect of LSM as compared to dobutamine. The haemodynamic effects of both drugs are similar; while LSM does not lead to an increased myocardial oxygen consumption, its arrhythmogenic influence is lower, and it exercises a positive effect combined with $\beta$-adrenolytic drugs [11-13]. The increase in SV and decrease in PCWP are greater for LSM than for dobutamine.

In 2005, Morelli et al. examined 28 patients with persistent dysfunction of the left ventricle, comparing two groups; group 1 received LSM, and group 2 received dobutamine. Better haemodynamic parameters were observed in the LSM group: increased CO, left ventricular work index (LVWI), and left ventricular ejection fraction (LVEF), as well as an increase in the supply and consumption of oxygen in the cells (DO2I, VO2I). There were significant decreases in PCWP, MPAP, and left ventricular enddiastolic volume (LVEDV). Other findings for the LSM group included an increase of daily diuresis, better visceral flow, and lower levels of lactates. No difference was observed for MAP, SVR, and PVR [14].

In 2010, Landoni et al. published results of a meta-analysis of randomised controlled trials (RCT) of 440 patients with heart failure. The effects of LSM and other inotropic drugs (milrinone, dobutamine) were compared. The study encompassed only patients with baseline low cardiac output operated on in cardiac surgery departments. Perioperative LSM treatment significantly decreased the mortality rate $(4.7 \%$ vs. $12.7 \%)$; lower levels of released troponin and lower frequency of arrhythmia were also observed [15].
The same year, an interesting study by Delaney et al. was published. It was based on the analysis of 3650 patients from 19 randomised controlled trials. The study encompassed patients with acute heart failure (HF : EF $<30-40 \%, \mathrm{Cl}<2.2-2.5$, NYHA III/IV, PCWP > 15). Comparing the use of LSM, dobutamine, and placebo, the following conclusions were formulated: no significant reduction of mortality rate in the LSM group vs. the placebo group; a considerable reduction of mortality rate in the LSM group vs. the dobutamine group; and a significant haemodynamic improvement and reduction of brain natriuretic peptide in serum in the LSM group in comparison to the other groups. In the conclusions of the study it was stated that further research was necessary to determine whether dobutamine increased mortality in patients with acute heart failure [16].

\section{Levosimendan in cardiac surgery}

In 2012, Landoni et al. provided results of another comprehensive meta-analysis (5400 patients in 45 RCTs). The study included patients with low cardiac output syndrome, treated in cardiology and cardiac surgery departments. The group given LSM was compared with the groups given: milrinone, enoxymon, prostaglandin E1 (PGE1), dobutamine, and placebo. Apart from improvement in haemodynamic parameters, a significantly lower mortality rate (17.5\% vs. $23.3 \%)$ and a shorter hospital stay were found in the LSM group [17].

In 2013, an expert consensus on perioperative LSM treatment in cardiac surgery was published. According to the recommendations, the indications for such a treatment include the following: low cardiac output syndrome, circulation decompensation, failure to come off extracorporeal circulation, and qualification for mechanical circulatory assistance. Before receiving LSM, patients should have optimum intravascular volume status, good acid-base balance and electrolyte balance, their BP should be constantly monitored, and they should receive add-on norepinephrine treatment in the case of low systolic BP (lower than $90 \mathrm{~mm} \mathrm{Hg}$ ). It is also recommended that $\beta$-adrenolytic treatment be continued and diuretic treatment be optimised. The authors of the consensus statement emphasise the need to be careful when applying the loading dose; they recommend avoiding bolus and, instead, administering LSM in a 24-hour non-stop infusion from $0.1 \mu \mathrm{g} / \mathrm{kg} / \mathrm{min}$ to $0.2 \mu \mathrm{g} /$ $\mathrm{kg} / \mathrm{min}$. The effects of the starting dose are visible after about $2 \mathrm{~h}$, the flow rate of the infusion has to be adjusted to the haemodynamic effects (increased or decreased by $0.05 \mu \mathrm{g} / \mathrm{kg} / \mathrm{min}$ ) [18].

In 2014, an expert consensus on the repetitive LSM therapies in cardiology was published. It concerns patients with chronic advanced heart fail- 
ure. The experts determined a group of patients who can benefit from repetitive administration of LSM. The group encompasses patients with acute systolic heart failure (LVEF < 35\%) and/or NYHA IIb-IV, after two or more hospitalisations in 1 year, and no effect from classical ADHF therapy. The recommended LSM doses do not differ from other doses, but they have to be administered every 2-4 weeks. The recommendations are based on nine clinical studies including about 500 patients. The patients repetitively taking LSM were found to have improved haemodynamic parameters and clinical conditions, fewer hospitalisations, and lower biochemical markers of myocardial damage. The experts emphasise the necessity to conduct further studies assessing the long-term effects of LSM treatment and its influence on mortality [10].

Other authors also proved the effectiveness of LSM in treating ADHF and confirmed that it caused a drop in the mortality rate in comparison to other commonly used medications $[19,20]$.

\section{Levosimendan in right-heart failure}

There are also reports confirming the effectiveness of LSM in right ventricular heart failure. LSM was proven to dilate pulmonary vessels, at the same time lowering right ventricular afterload. As a result, contractility of the right ventricle improves, PCWP drops, and right coronary artery flow increases [21].

Barisin et al. published an interesting case study of a patient with right ventricular acute heart failure in the early post-transplant period. Having applied classically used drugs (such as noradrenaline, dobutamine, isoprenaline, and nitro-glycerine), with deteriorating function of the graft and symptoms of right ventriclar acute heart failure, it was decided to introduce an LSM infusion as an emergency treatment. A 48-hour LSM infusion was used, bringing about a significant improvement, i.e. an increase in $\mathrm{CO}$, right ventricular stroke work index (RVSW), and central venous oxygen saturation, which led to improvement in the patient's condition [22].

Russ et al. proved the effectiveness of LSM in treating cardiogenic shock related to acute myocardial infarction and right ventricular heart failure. In the group of 56 patients a significant increase of $\mathrm{CO}$, and right ventricular cardiac power index, as well as a decrease of PVR were observed following administration of LSM [23].

\section{Levosimendan in takotsubo, post-partum cardiomyopathy, and stunned myocardium}

There are reports confirming beneficial effects of LSM use in peripartum cardiomyopathy and takotsubo cardiomyopathy.
It is commonly believed that myocardial damage leading to takotsubo cardiomyopathy is caused by high levels of catecholamines in serum induced by stress. It seems thus that the use of traditional inotropic drugs may not be appropriate here. LSM seems to be a good alternative, which improves heart function without an increase in catecholamine concentration.

This finds its confirmation in research conducted by Santoro et al., who described improvement in circulatory function in 13 patients who had been earlier treated with no effect with the use of traditional inotropic drugs. After administration of LSM, a significant increase of CO was observed, in line with a decrease in troponin levels. What is more, stenocardiac complaints subsided and therapeutic effects continued after discharging the patients from hospital [24].

In the literature there are more and more reports analysing the use of LSM in the case of neurogenically stunned myocardium after aneurysmal subarachnoid haemorrhage (aSAH) [25-27]. Its development is also dependent on stress-induced ejection of endogenous catecholamines, which leads to AHF symptoms. The clinical manifestation of the syndrome assumes the form of left ventricular heart failure, which leads to decreased cardiac output [28]. The characteristic triad of symptoms comprises: left ventricular contractility disorders, abnormal ECG (prolongation of QT interval, T-wave inversion, ST segment abnormalities), and an increased level of heart enzymes with no changes in coronary arteries [25]. It is believed that every abrupt increase in intracranial pressure may lead to development of neurogenic stunned myocardium, not as a result of increased catecholamines levels in blood, but rather as a result of increased personal/individual sensitivity of adrenergic receptors. Also, the generalised inflammatory response with proinflammatory cytokines and free radicals is likely to have some impact on the development of this condition [29].

An increased inflow of calcium ions into cardiomyocytes is responsible for decompensation of circulation, usually within $48 \mathrm{~h}$ of the activity of the trigger. Arrhythmia is a clinically common manifestation of this syndrome. Classical treatment of this type of circulatory failure can be ineffective not only because of potential adverse effects of catecholamines, but also because of lowering the sensitivity of $\beta 1$-adrenergic receptors (the so-called down-regulation) [25].

Taccone et al. presented a case study of a patient with aSAH and stunned myocardium symptoms in whom classical treatment (dobutamine, milrinone) was ineffective. After introducing LSM, a significant improvement in circulation was achieved; however, the patient died as a result of central nervous system damage [30]. 
Two other cases of LSM's positive impact in aSAH were described by Papanikolaou et al. As well as improvement in haemodynamic parameters and organ perfusion, the authors focused on the importance of stabilising the cerebral perfusion pressure after introducing LSM into the therapy. The authors also emphasise that they implemented a dose regimen without a loading dose [26] for fear of possible hypotension because it could have a really detrimental impact on the cerebral perfusion pressure.

Because stress-induced myocardial damage is the common denominator of the above presented cardiomyopathies, more and more researchers are inclined to believe that they are all clinical manifestations of the same syndrome. Some of the researchers think that this extends also to cardiomyopathy in life-threatening conditions and pheochromocytoma-related cardiomyopathy [29].

Symptoms of heart failure, cardiogenic shock in their extreme form, can appear in the course of peripartum cardiomyopathy (PPCM). This term denotes heart failure in the form of dilated cardiomyopathy, which refers to women during delivery or in the last month of pregnancy. The causes of this condition are not entirely known. It is thought that it originates from haemodynamic changes related to pregnancy; it may assume the form of myocardial inflammation caused by autoimmune aggression, infection, or gene-related changes. Treatment of PPCM is based on symptoms and does not differ from standard procedures in AHF, and the mortality rate ranges from $30 \%$ to $60 \%$ [31].

A few authors emphasise the positive impact of LSM also in this type of cardiomyopathy. In the available publications, there are a dozen or so case studies reporting significant improvement in haemodynamic parameters, subsidence of HF symptoms, and normalisation of left ventricle function [32, 33].

\section{Levosimendan and intoxication with calcium channel blockers}

Another interesting and crucial issue is the applicability of LSM in CCB toxicity. The basic pathomechanism of this intoxication consists of hindering calcium flow to cardiomyocytes, which results in acute myocardial failure, and, in many cases, in cardiogenic shock. CBB toxicity treatment focuses on symptoms and encompasses prevention of $a b$ sorption of further drug doses, administration of traditional inotropic drugs, calcium preparations, glucagon, and, in the most severe cases, introduction of intra-aortic counterpulsation. Methods of extracorporeal toxicity elimination are ineffective; there is no pharmacological remedy.

When it comes to LSM activity mechanism, it seems that it can reverse the effect of calci- um channel antagonists through increased flow of calcium ions to myocardial cells, irrespective of blocking their receptors. One should thus expect haemodynamic improvement in the form of increased strength of myocardial contraction, cardiac output, and, as a consequence, blood pressure.

Reports in the literature concerning this issue are still infrequent, but those that are available are mostly promising. Osthoff et al. presented a case of a verapamil-intoxicated patient who was given LSM because high doses of catecholamines did not bring any improvement [34]. A similar case was described by Varpula et al. [35]. Teker et al. reported a beneficial effect of LSM in amlodipine toxicity [36]. According to researchers, when traditional treatment of CCB toxicity fails, it is necessary to introduce LSM.

However, there are also contrary reports. For instance, Abraham et al. based their report on the animal model of verapamil toxicity [37]. The authors did not find increased survival in rats given LSM in comparison to the placebo group.

In both cases, confirming and disproving the effectiveness of LSM, the researchers emphasised the necessity to conduct further studies in this field.

\section{Levosimendan in sepsis}

One of the first reports on LSM is from 2001 and describes septic shock [38]. Sepsis causes depression of both heart ventricles through impaired response of $\beta$-receptors to endo- and exogenous catecholamines and through lowered sensitivity of myofilaments to calcium. When it comes to the pathophysiology of septic shock, LSM can be instrumental in improving the circulatory function.

A report from 2001 referred to research conducted on animals (swine) in which septic shock was induced by administering endotoxin Escherichia coli. In 14 cases a considerable improvement in haemodynamic parameters was observed (increased CO, decreased PCWP, PVR, MPAP); the function of some organs also improved (more oxygen was delivered to the heart and intestinal mucosa, diuresis improved, the level of blood lactates dropped) [38].

Similar research was undertaken by Rehberg on the sheep model of sepsis, with a significant improvement in left ventricular stroke work index (LVSWI) and right ventricular stroke work index (RVSWI), as well as a significant increase of central venous oxygen saturation, with decreases in PCWP, SVRI, and PVRI [39].

Among the latest LSM clinical programmes is LeoPARDS (Levosimendan for the Prevention of Acute Organ Dysfunction in Sepsis). The results of this study were published in October 2016. Its aim was to assess the effect of LSM on mul- 
tiple organ failure and mortality in septic shock. The study included 516 patients divided into two groups, the LSM group and the placebo group (in which septic shock was treated in line with generally accepted guidelines).

In the course of the study LSM was not proved to show benefits in septic shock treatment. The dysfunction of organs or the mortality did not diminish in the LSM group. Supraventricular tachyarrhythmias and the period of mechanical ventilation were longer in the LSM group. Patients in this group required a higher dose of noradrenaline in order to maintain the adequate RR. The effect on multiple organ failure was assessed with the SOFA (Sequential Organ Failure Assessment) scale from the $1^{\text {st }}$ to the $28^{\text {th }}$ day of the hospital stay. The SOFA scale helps assess five main organs: the heart with circulation system, lungs with respiratory system, kidneys, liver, and the coagulation system. In the LSM group, no improvement in organ function was found, as in the case of the abovementioned studies. The authors attribute the higher frequency of tachyarrhythmias in the LSM group to a higher supply of norepinephrine in this group (LSM does not stimulate $\beta$-receptors). A few limitations that might have influenced results of the study were also pointed out. One of them is comparing LSM to a standard therapy, not to an alternative inotropic drug, e.g. dobutamine. The use of dobutamine in the placebo group was higher than in the LSM group, which can explain why no greater haemodynamic benefits were found in the LSM group. The echocardiographic analysis was not applied as a method in the study in order to compare changes in myocardium function. It was also mentioned that the MAP (65$70 \mathrm{~mm} \mathrm{Hg}$ ) recommended in the study was often exceeded, so the dose of norepinephrine should have been reduced more often [40].

\section{Levosimendan - effects on an organ system other than the heart}

Heart failure often co-occurs with kidney failure. One of the symptoms of worsening ADHF is retention of fluids, in extreme cases leading to pulmonary oedema. Improvement in cardiac output, as well as decrease in preload and afterload, indirectly lead to an improvement in the function of kidneys and other organs (such as the liver, brain, and intestines). Some studies showed improvements in liver function on the basis of a drop in the level of liver aminotransferases and alkaline phosphatase [41-43].

One of the studies conducted on 44 patients after heart transplantation showed a statistically significant improvement in kidney parameters: a lower level of creatinine and better creatinine clearance. The so-called renoprotective effect per- sisted in some patients for 3 months after the end of the LSM treatment [44].

A direct effect of LSM on kidney blood vessels was also observed, namely LSM led to improvement in blood flow through kidneys [45]. Other studies confirmed the effectiveness of LSM in improving kidney function $[46,47]$.

On the basis of one of the retrospective meta-analyses, it was noticed that there were fewer complications of acute kidney injury after cardiac surgical procedures in groups that were given LSM, in comparison with the placebo, milrinone, and dobutamine groups [48].

One of the studies proved that early introduction of LSM (just after induction of anaesthesia before coronary artery bypass grafting) is correlated with better postoperative kidney function, when compared with later introduction of the medicine [49].

Positive effects of LSM are also visible in the central nervous system. An increased cardiac output leads to an increase in the cerebral blood flow, making it possible to avoid the application of exogenous catecholamines. There are reports on a direct neuroprotective effect of LSM related to its pleiotropic activity. LSM lowers the level of proinflammatory mediators (IL-6, TNF- $\alpha$ ) in the brain tissue, changing the area of its damage [25].

\section{Side effects}

The literature on the subject reports the following effects of LSM treatment: tachycardia, hypotension, heart arrhythmia, hypokalaemia, headache and vertigo, insomnia, and gastrointestinal disorders.

In the summary of product characteristics, LSM is not recommended for application in patients with hypotony, tachycardia, arrhythmia, and uncorrected hydro-electrolyte imbalances. In clinical practice, it is difficult to meet an ADHF patient without the above haemodynamic disorders. Most researchers recommend careful application of LSM in such cases, after correcting hypovolaemia and hypokalaemia, and achieving a systolic blood pressure above $100 \mathrm{~mm} \mathrm{Hg}$. During the LSM treatment it is recommended to use advanced haemodynamic monitoring, with special attention paid to the hydration of the patients, as well as $\mathrm{CO}$ and SVR. This allows the treatment to be complemented with other catecholamines (e.g. noradrenaline) when the BP drops. It is recommended that the level of electrolytes and the level of blood creatinine are monitored. It is not recommended that LSM be used in patients with significantly impaired kidney and liver function. No parameters determining the level of liver damage that could function as a counterindication for LSM use have so far been found in the literature. Such parame- 
ters are established for kidneys (GFR $<30 \mathrm{ml} / \mathrm{min}$ ). However, as demonstrated above, LSM can effectively improve the function of different organs by improving their perfusion; thus, every case ought to be treated individually [8].

\section{Conclusions}

The latest guidelines of the European Society of Cardiology (ESC) on treating acute and chronic heart failure state that LSM is classified as a IIB, level $C$ drug. Experts recommend LSM therapy for patients with severe reduction of cardiac output and organ perfusion, after eliminating hypovolaemia and other potential causes. LSM is preferred over dobutamine if patients also take $\beta$-blockers, which can be related to hypoperfusion. ESC emphasises the vasodilatative properties of LSM and recommends that clinicians are careful when it comes to patients with hypotonia and cardiogenic shock, and in such cases combine LSM with other inotropic drugs and medicines constricting vessels. The low class of recommendations results from doubts connected with the impact of LSM (and other inotropic drugs) on increasing mortality [1].

Summarising, it can be said that beneficial effects of LSM in improving circulation capacity in ADHF as well as in perioperative treatment in cardiac surgery have been confirmed in numerous studies. A few preclinical and clinical studies proved also a beneficial effect of LSM in septic shock, cardiomyopathies of various aetiologies, and toxicity of cardiodepressive drugs. However, the beneficial effect of LSM on the function of organs and lowering mortality in septic shock was not confirmed in the LeoPARDS study. Some authors mention inconsistent selection of patients in a few of the discussed studies, and a lot of publications conclude with the statement that further studies in this field are required.

\section{Conflict of interest}

The authors declare no conflict of interest.

\section{References}

1. Ponikowski P, Voors AA, Anker SD, et al. 2016 ESC Guidelines for the diagnosis and treatment of acute and chronic heart failure. The Task Force for the diagnosis and treatment of acute and chronic heart failure of the European Society of Cardiology (ESC). Developed with the special contribution of the Heart Failure Association (HFA) of the ESC. Eur Heart J 2016; 37: 2129-200.

2. Landoni G, Biondi-Zoccai G, Greco M, et al. Effects of levosimendan on mortality and hospitalization. A metaanalysis of randomized controlled studies. Crit Care Med 2012; 40: 63

3. Lilleberg J, Laine M, Palkama T, Kivikko M, Pohjanjousi P, Kupari M. Duration of the haemodynamic action of a 24-h infusion of levosimendan in patients with congestive heart failure. Eur J Heart Fail 2007; 9: 75-82.

4. Perrone SV, Kaplinsky EJ. Calcium sensitizer agents: a new class of inotropic agents in the treatment of decompensated heart failure. Int J Cardiol 2005; 103: 248-55.

5. Papp Z, Edes I, Fruhwald S, et al. Levosimendan: molec ular mechanisms and clinical implications: consensus of experts on the mechanisms of action of levosimendan. Int J Cardiol 2012; 159: 82-7.

6. Nieminem MS, Akkila J, Hasenfuss G, et al. Haemodynamic and neurohumoral effects of continous infusion of levosiemndan in patients with congestive heart failure. J Am Coll Cardiol 2000; 36:1903-12.

7. Kivikko M, Antila S, Eha J, Lehtonen L, Pentikäinen PJ. Pharmacodynamics and safety of a new calcium sensitizer, levosimendan, and its metabolites during an extended infusion in patients with severe heart failure. J Clin Pharmacol 2002; 42: 43-51.

8. Nieminem MS, Altenberger J, Ben-Gal T, et al. Repetetive use of levosimendan for treatment of chronic advanced heart failure: clinical evidence, practical consideration, and perspectives: an expert panel consensus. Int J Cardiol 2014; 174: 360-7.

9. Bayram M, de Luca L, Massie MB, et al. Reassessment of dobutamine, dopamine and milrinone in the management of acute heart failure syndromes. Am J Cardiol 2005; 96: 47G-58G.

10. Tackray S, Easthaug J, Freemantle N, et al. The effectiveness and relative effectiveness of intravenous inotropic drugs acting trough the adrenergic pathway in patients with heart failure-a meta-regression analysis. Eur J Heart Fail 2002; 4: 515-29.

11. Huang X, Lei S, Zhu MF, et al. Levosimendan versus dobutamine in critically ill patients: a meta-analysis of randomized controlled trials. J Zhejiang Univ Sci B 2013; 14: 400-15

12. Bergh $\mathrm{CH}$, Anderson B, Dahlstrom U, et al. Intravenous levosimendan vs.dobutamine in acute decompensated heart failure patients on beta-blockers. Eur J Heart Fail 2010; 12: 404-10.

13. Follath F, Cleland JG, Just H, et al. Efficacy and safety of intravenous levosimendan compared with dobutamine in severe low-output heart failure (the LIDO study): a randomised double-blind trial. Lancet 2002; 360: 196-202.

14. Morelli A, De Castro S, Teboul JL, et al. Effects of levosiemndan on systemic and regional hemodynamics in septic myocardial depression. Intensive Care Med 2005; 31: 638-44

15. Landoni G, Mizzi A, Biondi-Zoccai G, et al. Levosimendan reduces mortality in critically ill patients. A meta-analysis of randomized controlled studies. Minerva Anestesiol 2010; 76: 276-86.

16. Delaney A, Bradford C, Mc Caffrey J, et al. Levosimendan for the treatment of acute severe heart failure: a metaanalysis of randomised controlled trials. Int J Cardiol 2010; 138: 281-9.

17. Landoni G, Biondi-Zoccaai G, Greco M, et al. Effects of levosimendan on mortality and hospitalization. A metaanalysis of randomized controlled studies. Crit Care Med 2012; 40: 634-46.

18. Toller W, Algotsson L, Guarrancino F, et al. Perioperative use of levosimendan: best practice in operative settings. J Cardiothorac Vasc Anesth 2013; 27: 361-6.

19. Silvetti S, Greco T, Prima AL, et al. Intermittent levosimendan improves mid-term survival in chronic heart 
failure patients: meta-analysis of randomised trials. Clin Res Cardiol 2014;103: 505-13.

20. Poelzl G. Efficacy and safety of intermittent ambulatory infusion of levosimendan in end-stage heart failure. At Special Session, Latest Updates/Heart Failure 2013 Congress of the ESC-HFA. Lisbon, May 2013. http:// spo.escardio.org/SessionDetails.aspx? eevtid $=61$ \& sessld $=10924 \&$ subSessld $=0$. accessed 17 October, 2013.

21. Westphal M, Morelli A, Van Aken H. Dear levosimendan, the right ventricule will thank you! Crit Care Med 2007 35: 952-3.

22. Barisin S, Djuzel V, Barisin A, Rudez I. Levosimendan reverses right-heart failure in a 51 -year-old patient after heart transplantation. Wien Klin Wochenschr 2014 126: 495-9.

23. Russ MA, Prondzinsky R, Carter JM, et al. Right ventricular function in myocardial infarction complicated by cardiogenic shock: improvement with levosimendan. Crit Care Med 2009; 37: 3017-23.

24. Santoro F, Leva R, Ferraretti A, et al. Safety and feasibility of levosimendan administration in takotsubo cardiomy opathy; a case series. Cardiovasc Ther 2013; 31: e133-7.

25. Varvarousi G, Xanthos T, Sarafidou P, et al. Role of levosimendan in the management of subarahnoidal hemorrhage. Am J Emerg Med 2016; 34: 298-306.

26. Papanikolaou J, Tsolaki V, Makris D, et al. Early levosimendan administration may improve outcome in patients with subarachnoid hemorrhage complicated by acute heart failure. Int J Cardiolo 2014; 176: 1435-7.

27. Busani S, Rinaldi L, Severino C, et al. Levosimendan in cardiac failure after subarachnoid hemorrhage. J Trauma 2010; 68: E108-10.

28. Papanikolaou J, Makris D, Karakitsos D, et al. Cardiac and central vascular functional alternations in the acute phase of aneurysmal subarachnoid hemorrhage. Crit Care Med 2012; 40: 223-32.

29. Mierzewska-Schmidt M, Gawecka A. Neurogenic stunned myocardium-do we consider this diagnosis in the patients with acute central nervous system injury and acute heart failure? Anaesthesiol Intensive Ther 2015; 47: 175-80.

30. Taccone FS, Brasseur A, Vincent JL, De Backer D. Levosimendan for the treatment of subarachnoid hemorrhage-related cardiogenic shock. Intensive Care Med 2013; 39: 1497-8

31. Uriarte-Rodriguez A, Santana-Cabrera L, Sanchez-Palacios $M$. Levosimendan use in the emergency management of decompensated peripartum cardiomyopathy. J Emerg Trauma Shock 2010; 3: 94.

32. Benlolo S, Lefoll C, Katchatouryan V, et al. Successful use of levosimendan in a patient with peripartum cardiomyopathy. Anesth Analg 2004; 98: 822-4.

33. Benezet-Mazuecos J, de la Hera J. Peripartum cardiomyopathy: a new successful setting for levosimendan. Int J Cardiol 2008; 123: 346-7.

34. Osthoff M, Bernsmeier C, Marsch SC, Hunziker PR. Levosimendan as treatment option in severe verapami intoxication: a case report and review of the literature. Case Rep Med 2010; 2010, pii: 546904.

35. Varpula T, Rapola J, Sallisalmi M, et al. Treatment of serious calcium channel blocker overdose with levosimendan, a calcium sensitizer. Anesth Analg 2009; 108 790-2.

36. Teker MG, Ozdemir H, Saidoglu L, et al. Levosimendan as a rescue adjunct in amlodipine intoxication - a case report. Middle East J Anaesthesiol 2010; 20: 869-72.
37. Abraham MK, Scott SB, Meltzer A, et al. Levosimendan does not improve survival time in a rat model of verapamil toxicity. J Med Toxicol 2009; 5: 3-7.

38. Oldner A, Konrad D, Weitzberg E, et al. Effects of levosimendan, a novel inotropic calcium-sensitizing drug, in experimental septic shock. Crit Care Med 2001; 29: 2185-93.

39. Rehberg S, Ertmer C, Vincent JL, et al. Effects of combined arginine vasopressin and levosimendan on organ function in ovine septic shock. Crit Care Med 2010; 38: 2016-23.

40. Gordon AC, Perkins GD, Singer M, et al. Levosimendan for the prevention of acute organ dysfunction in sepsis. N Engl J Med 2016; 375: 1638-48.

41. Nikolaou M, Parissis J, Yilmaz MB, et al. Liver function abnormalities, clinical profile and outcome in acute decompensated heart failure. Eur Heart J 2013; 34: 742-9.

42. Grossini E, Pollessello P, Bellofatto K, et al. Protective effects elicited by levosimendan against liver ischemia/ reperfusion injury in anesthetized rats. Liver Transpl 2014; 20: 361-75.

43. Memis D, Inal MT, Sut N. The effects of levosimendan vs dobutamine added to dopamine on liver functions assessed with noninvasive liver function monitoring in patients with septic shock. J Crit Care 2012; 27: 318.e1-6.

44. Zemljic G, Bunc M, Yazdanbakhsh AP, Vrtovec B. Levosimendan improves renal function in patients with advanced chronic heart failure awaiting cardiac transplantation. J Card Fail 2007; 13: 417-21.

45. Bragadottir G, Redfors B, Ricksten SE. Effests of levosimendan on glomerular filtration rate, renal blood flow, and renal oxygenation after cardiac surgery with cardiopulmonary bypass: a randomized placebo-controlled study. Crit Care Med 2013; 41: 2328-35.

46. Yilmaz MB, Grossini E, Silva Cardoso JC, et al. Renal effects of levosimendan: a consensus report. Cardiovasc Drugs Ther 2013; 27: 581-90.

47. Fedele F, Bruno N, Brasolin B, Caira C, D’Ambrosi A, Mancone M. Levosimendan improves renal function in acute decompensated heart failure: possible underlying mechanisms. Eur J Heart Fail 2014; 16: 281-8.

48. Niu ZZ, Wu SM, Sun WY, Hou WM, Chi YF. Perioperative levosimendan therapy is associated with a lower incidence of acute kidney injury after cardiac surgery: a meta-analysis. J Cardiovasc Pharmacol 2014; 63: 107-12.

49. Balzer F, Treskatsch S, Spies C, et al. Early administration of levosimendan is associated with improved kidney function after cardiac surgery - a retrospective analysis. J Cardiothorac Surg 2014; 9: 167. 